MADPH-95-875

University of Wisconsin - Madison

RAL-95-026

OUTP-95-09P

hep-ph/9503295

April 1995

\title{
Remarks on the KARMEN anomaly
}

\author{
V. Barger ${ }^{a}$, R.J.N. Phillips ${ }^{b}$ and S. Sarkar ${ }^{c}$ \\ ${ }^{a}$ Physics Department, University of Wisconsin, Madison, WI 53706, USA \\ ${ }^{b}$ Rutherford Appleton Laboratory, Chilton, Didcot, Oxon OX11 OQX, UK \\ ${ }^{c}$ Theoretical Physics, University of Oxford, 1 Keble Road, Oxford OX1 3NP, UK
}

\begin{abstract}
A recently reported anomaly in the time structure of signals in the KARMEN neutrino detector suggests the decay of a new particle $x$, produced in $\pi^{+} \rightarrow \mu^{+} x$ with mass $m_{x}=33.9 \mathrm{MeV}$. We discuss the constraints and difficulties in interpreting $x$ as a neutrino. We show that a mainly-sterile neutrino scenario is compatible with all laboratory constraints, within narrow limits on the mixing parameters, although there are problems with astrophysical and cosmological constraints. This scenario predicts that appreciable numbers of other $x$-decay events with different origins and time structures should also be observable in the KARMEN detector. Such $x$-decay events should also be found in the LSND experiment and may be relevant to the search for $\bar{\nu}_{\mu} \rightarrow \bar{\nu}_{e}$ oscillations.
\end{abstract}

(Published in Phys. Lett. B352 (1995) 365; erratum added June 1995) 
The KARMEN collaboration, which studies the interactions of neutrinos from the stopped $\pi^{+}$decay chain at RAL, has recently reported an anomaly [1] in the timedependence of their signals. This anomaly suggests the production of a new weaklyinteracting neutral particle (call it $x$ ) in the initial $\pi^{+}$decays, which travels with well determined velocity $\beta_{x}=v_{x} / c \simeq 1 / 60$ and decays in the detector after a mean flight path of $17.5 \mathrm{~m}$. The distinctive feature of the $x$-events is their timing, apparently at a well determined interval $3.6 \mu$ s after the arrival and prompt decay of the pion pulse (which determines $\beta_{x}$ ); however, the visible energy in the detector scintillator shows no anomaly, so $x$ decays apparently deposit visible energy similar to typical neutrino interactions. The present note briefly discusses the interpretation of $x$ as a massive neutrino. We show that a mainly-sterile neutrino scenario is compatible with all laboratory constraints, for either Dirac or Majorana options, within rather narrow bounds on the mixing parameters. There are some problems with astrophysical and cosmological constraints, but it is interesting nevertheless to explore the further implications for laboratory experiments which can test this interpretation directly. This scenario predicts that appreciable numbers of other $x$-decay events with different origins and time structures should also be observable in the KARMEN detector. Such $x$-decay events should also be found in the LSND experiment at LAMPF [2] and may be relevant to the ongoing search for $\bar{\nu}_{\mu} \rightarrow \bar{\nu}_{e}$ oscillations 3 .

If we postulate no other new particles below the pion mass, then the precise time structure [1] requires $x$ production to go via one of the two-body modes $\pi^{+} \rightarrow \mu^{+} x$ or $\pi^{+} \rightarrow e^{+} x$. However the latter implies mass $m_{x}=137.2 \mathrm{MeV}$ (determined from $\beta_{x}$ ) and hence anomalously large visible $x$-decay energy, with mean value $\left\langle T_{\text {vis }}>\simeq 51\right.$ $\mathrm{MeV}$ for $x \rightarrow \mu e \nu \rightarrow e e \nu \nu \nu$ or $<T_{\text {vis }}>\simeq 88 \mathrm{MeV}$ for $x \rightarrow e e \nu$, compared to neutrino interactions that typically give $T_{\text {vis }} \sim 11-35 \mathrm{MeV}$ [1]. We must therefore presume that $x$ is produced via $\pi^{+} \rightarrow \mu^{+} x$, with $m_{x}=33.9 \mathrm{MeV}$ determined from $\beta_{x}$. Assuming no new weak interactions, the standard decay mode is $x \rightarrow e^{-} e^{+} \nu_{e}$; note that flavour-changing neutral current (FCNC) processes $x \rightarrow \nu \nu \nu$ and $x \rightarrow \nu \gamma$ are highly suppressed for a standard isodoublet neutrino (we consider the isosinglet case later) [4. [5].

The neutrino charged-current eigenstates $\nu_{\alpha \mathrm{L}}(\alpha=e, \mu, \tau)$ which appear in the weak interaction coupled via $W$ to $e, \mu, \tau$, may be written as coherent superpositions of mass 
eigenstates $\nu_{i \mathrm{~L}}(i=1,2, \ldots x)$ using the usual mixing matrix $U_{\alpha i}$;

$$
\nu_{\alpha \mathrm{L}}=\Sigma_{i} U_{\alpha i} \nu_{i \mathrm{~L}}
$$

(In general $U$ is a $n \times n$ matrix, with $\alpha$ running over all $S U(2)_{L}$ multiplet assignments and $i$ running over all masses). Then the $x$ production and decay processes are scaled by factors $\left|U_{\mu x}\right|^{2}$ and $\left|U_{e x}\right|^{2}$, respectively:

$$
\begin{aligned}
\frac{\Gamma(\pi \rightarrow \mu x)}{\Gamma(\pi \rightarrow \mu \nu)} & =\frac{\left|U_{\mu x}\right|^{2}\left[m_{\pi}^{2}\left(m_{\mu}^{2}+m_{x}^{2}\right)-\left(m_{\mu}^{2}-m_{x}^{2}\right)^{2}\right] \lambda^{\frac{1}{2}}\left(m_{\pi}^{2}, m_{\mu}^{2}, m_{x}^{2}\right)}{m_{\mu}^{2}\left(m_{\pi}^{2}-m_{\mu}^{2}\right)^{2}}, \\
\frac{\Gamma\left(x \rightarrow e^{+} e^{-} \nu_{e}\right)}{\Gamma\left(\mu \rightarrow \nu_{\mu} \bar{\nu}_{e} e\right)} & =\frac{\left|U_{e x}\right|^{2} m_{x}^{5}}{m_{\mu}^{5}}\left[\text { Dirac] }, \frac{2\left|U_{e x}\right|^{2} m_{x}^{5}}{m_{\mu}^{5}}\right. \text { [Majorana], }
\end{aligned}
$$

where $\lambda(a, b, c)=a^{2}+b^{2}+c^{2}-2 a b-2 b c-2 c a$ and we neglect $m_{e}^{2} / m_{x}^{2}$; we recall that Majorana neutrinos decay twice as fast as Dirac neutrinos with the same coupling, because their right chiral components are not inert. Hence the production branching fraction and mean decay lifetime are simply given by

$$
\begin{gathered}
B(\pi \rightarrow \mu x)=0.0285\left|U_{\mu x}\right|^{2} \\
\tau(x \rightarrow e e \nu)=645\left|U_{e x}\right|^{-2} \mu \mathrm{s}\left[\text { Dirac] }, \quad \tau(x \rightarrow e e \nu)=323\left|U_{e x}\right|^{-2} \mu \mathrm{s}\right. \text { [Majorana] }
\end{gathered}
$$

The correlation between branching fraction and lifetime, needed to explain the KARMEN anomaly, was shown as a curve in the $(\tau, B)$ plane in Ref. [1]. We reproduce this as the solid curve in Fig.1, extrapolating along the dashed curve with fixed $B / \tau$, and showing also the scales of $\left|U_{e x}\right|^{2}$ and $\left|U_{\mu x}\right|^{2}$ implied by Eqs.(4)-(5) along the upper and righthand edges of the diagram (the Dirac option is illustrated for $\left|U_{e x}\right|^{2}$ ). The regions with $\left|U_{\alpha x}\right|^{2}>1$ have no physical meaning in our scenario.

Direct experimental constraints on the mixing elements $\left|U_{\alpha i}\right|$ are summarized in Ref. [6]; they generally depend on mass and the constraints we quote below are all for $m_{x}=33.9 \mathrm{MeV}$. Absence of a correction to the $\rho$ parameter of the $e$ spectrum in $\mu \rightarrow e \nu \nu$ decay gives [7]

$$
\left|U_{e x}\right|^{2}+\left|U_{\mu x}\right|^{2}<2 \times 10^{-3}
$$

Absence of decay events in neutrino beams gives [8, 9, 10]

$$
\left|U_{e x}\right|\left|U_{\mu x}\right|<1.5 \times 10^{-5}
$$

Absence of anomalous contributions to $\pi \rightarrow e \nu$ gives [11]

$$
\left|U_{e x}\right|^{2}<0.85 \times 10^{-6}
$$


Limits from neutrinoless $\beta \beta$-decay searches [12 on the effective $\nu_{e}$ Majorana mass $\left\langle m_{\nu e}\right\rangle=\left|\Sigma_{j} \eta_{j} m_{j} U_{e j}^{2}\right|$, where $\eta_{j}= \pm$ is the CP signature of Majorana neutrino $\nu_{j}$, would require (see also Ref. 13])

$$
\left.\left|U_{e x}\right|^{2} \lesssim 6 \times 10^{-8} \quad \text { [Majorana }\right]
$$

where we have conservatively taken $\left\langle m_{\nu e}\right\rangle<2 \mathrm{eV}$ [12]. This bound would apply if $x$ were a Majorana state and there were no substantial cancellations in the sum but not if $x$ were a Dirac neutrino or part of a quasi-Dirac pair (with opposite CP signatures). Other direct laboratory constraints are weaker than these [6]. Studies of short muon tracks in $\pi \rightarrow \mu \rightarrow e$ events, from pions stopping in emulsion, would give stringent constraints on $\left|U_{\mu x}\right|$ for $m_{x}<33 \mathrm{MeV}\left[7\right.$; but for the present value $m_{x}=33.9 \mathrm{MeV}$, the muon kinetic energy is only $1.5 \mathrm{keV}$ giving an unobservable track length less than 1 micron, so no constraint can be derived on this basis. The $x$ mass and mixing predict a contribution to the $\mu \rightarrow e \gamma$ branching fraction 14

$$
B(\mu \rightarrow e \gamma)=3 \alpha m_{x}^{2} /\left(32 \pi M_{W}^{2}\right)\left|U_{e x}^{*} U_{\mu x}\right|^{2} \simeq 2.5 \times 10^{-23}
$$

a factor $\sim 2 \times 10^{12}$ below the experimental upper limit [6]. The constraints of Eqs.(河)-(8) are shown on Fig.1; they leave a range of "solutions" to the KARMEN anomaly, based on $x \rightarrow e^{-} e^{+} \nu$ decay, described by

$$
\begin{aligned}
\left|U_{e x}\right|\left|U_{\mu x}\right| & \simeq 0.8 \times 10^{-6}[\text { Dirac }], \quad 0.6 \times 10^{-6} \text { [Majorana] } \\
\left|U_{\mu x}\right| & \lesssim 4.5 \times 10^{-2}, \\
\left|U_{e x}\right| & \lesssim 10^{-3}\left[\text { Dirac] }, 2.5 \times 10^{-4} \quad[\text { Majorana }]\right.
\end{aligned}
$$

There are also constraints on the mass and identification of $x$. The ARGUS bound $m\left(\nu_{\tau}\right)<31 \mathrm{MeV}$ [15], the CLEO bound $m\left(\nu_{\tau}\right)<32.6 \mathrm{MeV}$ [16] and the recent ALEPH bound $m\left(\nu_{\tau}\right)<24 \mathrm{MeV}$ [17] (all at 95\% C.L.), exclude $x$ from being the major component of $\nu_{\tau}$. Since LEP experiments measure $N_{\nu}=2.988 \pm 0.023$ [18] light neutrino species (weighted by their isodoublet mixing factors), the chiral component $x_{\mathrm{L}}$ participating in standard weak interactions must then be dominantly isosinglet, i.e. sterile.

An isodoublet interpretation of $x$ is also excluded by cosmological and astrophysical arguments concerning unstable neutrinos in the mass and lifetime range of interest [19]. If $x$ has standard weak interactions, its cosmological relic abundance would be sufficiently 
high that its decay products would have distorted the spectrum of the $2.73 \mathrm{~K}$ blackbody radiation background unless its lifetime were less than $\sim 10^{5} \mathrm{~s}$ [20,21]. This requires $\left|U_{e x}\right|^{2} \gtrsim 10^{-8}$ (for $m_{x}=33.9 \mathrm{MeV}$ ) and removes part of the solution range in Eq.(13). At such early times the background photons are energetic enough to be Compton scattered by the $e^{+} e^{-}$pairs from $x$ decay to energies above the ${ }^{2} \mathrm{H}$ photofission threshold and may thus undo nucleosynthesis [21]. Taking into account the energy degradation due to $\gamma-\gamma$ scattering [22] this sets a lifetime bound of $\lesssim 2 \times 10^{3}$ s 23 corresponding to $\left|U_{e x}\right|^{2} \gtrsim 5 \times 10^{-7}$, which leaves only a tiny region of the remaining solution range. (Further constraints on isodoublets from consideration of the entropy production by the decaying particle are rather sensitive to the adopted upper limit to the primordial ${ }^{4} \mathrm{He}$ abundance [24].) This loophole is closed by consideration of the production and decays of massive neutrinos in Supernova 1987A. For example the process $x \rightarrow \nu_{e} e^{+} e^{-} \gamma$ operates at a rate $\alpha / 2 \pi$ relative to the decay $x \rightarrow \nu_{e} e^{+} e^{-}$and would have generated a $\gamma$ ray burst which was not observed by the SMM satellite [25]. When combined with other arguments relating to energy deposition inside the supernova [26], as well as experimental bounds on fast $\nu_{\tau}$ decays [8,9], this rules out all lifetimes shorter than $\sim 10^{8} \mathrm{~s}$ [27], forbidding the entire solution range for doublet neutrinos.

In order to evade the above bounds, it may appear adequate to require $x$ to be mainly sterile since its direct production, both in supernovae and in the early universe, is then suppressed. However, sterile neutrinos can also be produced through their mixing with doublet neutrinos, modulated by matter effects. During the collapse phase of a supernova, resonant $\nu_{e} \rightarrow x$ conversions may cause rapid deleptonisation of the core which would probably prevent the supernova explosion [28]; however this does not happen for $\Delta m^{2} \gtrsim 10^{8} \mathrm{eV}^{2}$ so will not apply to the $x$ particle. Secondly the energy loss due to emission of sterile neutrinos during the cooling phase would have excessively shortened the $\overline{\nu_{e}}$ burst from SN 1987A for a mixing in the range $\left|U_{e x}\right|^{2} \sim 10^{-9}-10^{-2}$ [29]; this argument applies to a neutrino with mass up to $\sim 50-100 \mathrm{MeV}$ [30] (see also Ref. [13]) and will rule out the allowed region for $x$. Most crucially, the absence of a $\gamma$ ray burst from SN 1987A would, as in the case of doublet neutrinos discussed above, rule out radiative decays [30] with lifetimes in the range $\sim 10^{1}-10^{8} \mathrm{~s}$, taking into account the enhancement of radiative decays for a singlet neutrino (see below). The production of sterile neutrinos through neutrino oscillations in the early universe 31] also presents 
a problem since $x$ particles can be brought into thermal equilibrium at temperatures exceeding a few $\mathrm{GeV}$. Although the $x$ relic abundance is thus substantially diluted by the entropy release during the subsequent quark-hadron phase transition, $x$ decays with a lifetime $\gtrsim 0.1 \mathrm{~s}$ will have an adverse effect on primordial nucleosynthesis [32 (see also Ref. [13]). Thus the interpretation of the KARMEN anomaly as a singlet neutrino is severely challenged by astrophysical and cosmological arguments, although there may be a loophole for fast decays with lifetime $\ll 0.1 \mathrm{~s}$ which occur in the supernova core and, in the early universe, before nucleosynthesis. Therefore we proceed to examine the implications of this hypothesis for laboratory experiments which can test it definitively.

Identifying $x$ as mainly sterile has several distinct repercussions for the $x$-decay modes, since GIM cancellations no longer suppress FCNC:

(i) The $x \rightarrow \nu \nu \nu$ invisible modes are now appreciable; we obtain

$$
\Gamma(x \rightarrow \nu \nu \nu) / \Gamma\left(x \rightarrow e^{-} e^{+} \nu_{e}\right)=\left[\left|U_{e x}\right|^{2}+\left|U_{\mu x}\right|^{2}+\left|U_{\tau x}\right|^{2}\right] /\left|U_{e x}\right|^{2}
$$

assuming that just one singlet neutrino flavour takes part and the other 3 neutrinos are much lighter than $x$; thus the invisible branching fraction is never less than $50 \%$. But our determination of $\left|U_{e x}\right|^{2}$ from the KARMEN $B / \tau$ plot is preserved, because the latter is determined by the visible decays only: $\tau=\Gamma(x \rightarrow \text { vis })^{-1}$. Adding invisible decay modes increases the total $x$-width and hence the total number of decays in a given detector, but the visible fraction decreases by the same factor and the number of visible decays remains unchanged (so long as the distance to the detector remains much less than the mean decay length, which is the case here).

(ii) Radiative decays $x \rightarrow \nu \gamma$, going via loop diagrams, also escape GIM suppression for mainly-singlet $x$; we obtain [4,5]

$$
\Gamma(x \rightarrow \nu \gamma) / \Gamma(x \rightarrow \nu \nu \nu)=\frac{27 \alpha}{8 \pi}=\frac{1}{128},
$$

assuming one singlet and summing 3 light final flavours as before. Radiative decays are thus between $0.4 \%$ and $0.8 \%$ of total decays, but can dominate visible decays as follows:

$$
\begin{gathered}
\left|U_{\mu x}\right|^{2}+\left|U_{\tau x}\right|^{2}>127\left|U_{e x}\right|^{2}, \quad x \rightarrow \nu \gamma \text { dominates; } \\
\left|U_{\mu x}\right|^{2}+\left|U_{\tau x}\right|^{2}<127\left|U_{e x}\right|^{2}, \quad x \rightarrow e^{-} e^{+} \nu \text { dominates. }
\end{gathered}
$$

(iii) When $x \rightarrow \nu \gamma$ modes start to dominate over $x \rightarrow e^{-} e^{+} \nu$ in the visible decays, we can no longer compensate an increase in $x$-production (increase in $\left|U_{\mu x}\right|^{2}$ ) by a decrease 
in $\left|U_{e x}\right|^{2}$, so we have to add Eq.(17) to the solution constraints for a sterile neutrino. This leaves a reasonable range for sterile Dirac but only a small region for sterile Majorana solutions in Fig.1.

(iv) A new class of solution is possible, in which $x \rightarrow \nu \gamma$ dominates the visible decays, in contrast to the solutions displayed in Fig.1 where $x \rightarrow e^{-} e^{+} \nu$ dominates. These solutions have $B \times \Gamma(x \rightarrow$ vis $) \simeq 3 \times 10^{-17} \mu \mathrm{s}^{-1}$ with $B$ determined by Eq.(4) as before, but $\left|U_{e x}\right|^{2}$ now contributes negligibly to the decay and we have $\Gamma(x \rightarrow$ vis $)=$ $1.2 \times 10^{-5}\left(\left|U_{\mu x}\right|^{2}+\left|U_{\tau x}\right|^{2}\right) \mu \mathrm{s}^{-1}$ for Dirac $x$ (double this for Majorana $x$ ). Hence these solutions are characterized by

$$
\left|U_{\mu x}\right|^{2}\left(\left|U_{\mu x}\right|^{2}+\left|U_{\tau x}\right|^{2}\right) \simeq 0.8 \times 10^{-10}\left[\text { Dirac] }, \quad 0.4 \times 10^{-10} \text { [Majorana }\right]
$$

which automatically satisfies the requirement $\left|U_{\mu x}\right|^{2}<2 \times 10^{-3}$ from Eq.(6). They are also constrained by the preliminary bound on $\nu_{\tau} \rightarrow \nu_{i} \gamma$ decays 33 from the BEBC-WA66 beam dump experiment [9], which here translates into

$$
\left|U_{\tau x}\right|^{2}\left(\left|U_{\mu x}\right|^{2}+\left|U_{\tau x}\right|^{2}\right) \lesssim 0.016[\text { Dirac] } \quad 0.008 \text { [Majorana] }
$$

The $x$ mean lifetime, dominated by $x \rightarrow \nu \nu \nu$ decays, can range between about $50 \mathrm{~s}$ (at the limit where $\left|U_{\mu x}\right|>>\left|U_{\tau x}\right|$ ) to about $5 \times 10^{-3} \mathrm{~s}$ (where $\left|U_{\tau x}\right|$ approaches its upper limit from Eq.(19)). Thus the $x$ mean decay length is always much greater than the distance to the KARMEN detector, as required. Also at the lower end of this lifetime range, the cosmological and astrophysical constraints may perhaps be evaded.

We now address the further implications of the $x$-neutrino scenario for laboratory experiments. This scenario implies that, with the stopped $\pi^{+}$decay chain as the neutrino source, $x$ will be produced not only via $\pi^{+} \rightarrow \mu^{+} x$ (giving the time-signatured anomaly in the KARMEN detector), but also via $\pi \rightarrow e^{+} x$ and $\mu^{+} \rightarrow \bar{\nu}_{\mu} x e^{+}, \bar{x} \nu_{e} e^{+}$channels. It is interesting to ask how many of these $x$ should decay in the KARMEN detector (or other detectors), compared to the anomaly events, and what their signatures may be.

(a) The $\pi^{+} \rightarrow e^{+} x$ channel. The rate depends on $\left|U_{e x}\right|^{2}$. The fraction that decay in a given detector depends inversely on the momentum with which $x$ is produced. Hence the ratio of detected $x$ decays from this channel compared to "anomaly" events from $\pi^{+} \rightarrow \mu^{+} x$ is

$$
\frac{N(\pi \rightarrow e x: x \text { detected })}{N(\pi \rightarrow \mu x: x \text { detected })}=\frac{\left|U_{e x}\right|^{2}\left[m_{\pi}^{2}\left(m_{e}^{2}+m_{x}^{2}\right)-\left(m_{e}^{2}-m_{x}^{2}\right)^{2}\right]}{\left|U_{\mu x}\right|^{2}\left[m_{\pi}^{2}\left(m_{\mu}^{2}+m_{x}^{2}\right)-\left(m_{\mu}^{2}-m_{x}^{2}\right)^{2}\right]} \simeq 0.15 \frac{\left|U_{e x}\right|^{2}}{\left|U_{\mu x}\right|^{2}}
$$


In these events $x$ has velocity $\beta_{x}=0.89$ and reaches the detector essentially in coincidence with the prompt $\nu_{\mu}$ burst from the initial pion pulse, well within the resolution defined by the $100 \mathrm{~ns}$ pulse length at the KARMEN source. For solutions with dominant $x \rightarrow e^{-} e^{+} \nu$ visible decays, the spectrum of visible energy (summed $e^{+} e^{-}$kinetic energies) is shown in Fig.2, including initial $x$ polarization and folding in the relative decay probability in a detector. About $76 \%$ of such detected decays have visible energy greater than $40 \mathrm{MeV}$, whereas each prompt $\nu_{\mu}$ carries only $29.8 \mathrm{MeV}$ total energy, so these $x$-decay events should be quite distinctive. For the more restricted Majorana solutions, $\left|U_{e x}\right|^{2} /\left|U_{\mu x}\right|^{2} \lesssim 10^{-2}$ and the $\pi \rightarrow e x$ signal is strongly suppressed. Solutions with dominant $x \rightarrow \nu \gamma$ visible decays necessarily have $\left|U_{e x}\right|^{2}<<\left|U_{\mu x}\right|^{2}+\left|U_{\tau x}\right|^{2}$; if this is achieved with $\left|U_{\mu x}\right|^{2}<\left|U_{e x}\right|^{2}<<$ $\left|U_{\tau x}\right|^{2}$, then the $\pi \rightarrow e x$ production channel will be important, giving decay photon energies from 4 to $70 \mathrm{MeV}$ with mean energy $26 \mathrm{MeV}$, to be compared with a narrow spike at $E_{\gamma}=17 \mathrm{MeV}$ from anomaly events.

(b) The $\mu^{+} \rightarrow \bar{\nu}_{\mu} x e^{+}$channel. Once again the rate depends on $\left|U_{e x}\right|^{2}$ and the fraction decaying in a given detector depends inversely on the $x$-momentum. Numerical calculations give the detected event ratio:

$$
\frac{N\left(\pi^{+} \rightarrow \mu^{+} \rightarrow \bar{\nu}_{\mu} e^{+} x: x \text { detected }\right)}{N(\pi \rightarrow \mu x: x \text { detected })} \simeq 0.38 \frac{\left|U_{e x}\right|^{2}}{\left|U_{\mu x}\right|^{2}}
$$

There is a spread of velocities $0<\beta_{x}<0.95$, but these events are smeared anyway by the parent muon lifetime and arrive with essentially the same time distribution as the $\bar{\nu}_{\mu}$ and $\nu_{e}$ interaction events. The detected visible energy spectrum for solutions with dominant $x \rightarrow e^{-} e^{+} \nu$, calculated for the $\mu \rightarrow x \rightarrow e e \nu$ cascade with full spin-correlated decay matrix elements [34], is shown in Fig.2; it has mean value $<T_{\text {vis }}>\simeq 26 \mathrm{MeV}$. This spectrum roughly resembles the ${ }^{12} \mathrm{C}\left(\nu_{e}, e^{-}\right) X$ spectrum shown in Ref. [1] , but has a longer tail above $35 \mathrm{MeV}$, so it should be possible to distinguish one from the other. As with channel (a) above, this channel is suppressed by $\left|U_{e x}\right|^{2} /\left|U_{\mu x}\right|^{2} \lesssim 10^{-2}$ for Majorana $x \rightarrow e^{-} e^{+} \nu$ solutions, but is important for a subset of $x \rightarrow \nu \gamma$ solutions, for which the decay photon energies extend from 5 to $53 \mathrm{MeV}$ with mean value $26 \mathrm{MeV}$.

(c) The $\mu^{+} \rightarrow \bar{x} \nu_{e} e^{+}$channel. Here the rate depends on $\left|U_{\mu x}\right|^{2}$ instead, like the "anomaly" channel $\pi \rightarrow \mu x$. Numerical calculations give the detected event ratio:

$$
\frac{N\left(\pi^{+} \rightarrow \mu^{+} \rightarrow \bar{x} \nu_{e} e^{+} x: \bar{x} \text { detected }\right)}{N(\pi \rightarrow \mu x: x \text { detected })} \simeq 0.40 .
$$

As with channel (b), these events have essentially the same time distribution as the $\bar{\nu}_{\mu}$ 
and $\nu_{e}$ interaction events; on the other hand, the number of these events compared to time-anomaly events is now firmly predicted. The detected visible energy spectrum for $x \rightarrow e^{-} e^{+} \nu$ solutions is shown in Fig.2; like case (b) it has mean value $\left\langle T_{\text {vis }}>\simeq 26\right.$ $\mathrm{MeV}$ and can be distinguished from conventional ${ }^{12} \mathrm{C}\left(\nu_{e}, e^{-}\right) X$ by the tail above $35 \mathrm{MeV}$. For $x \rightarrow \nu \gamma$ solutions, the decay photon again has energies between 5 and $53 \mathrm{MeV}$ with mean value $28 \mathrm{MeV}$.

To summarize, a mainly-sterile neutrino interpretation for $x$ is consistent with all laboratory constraints, within limited ranges of mixing parameters, for both Dirac and Majorana options, although there are some astrophysical and cosmological problems. Solutions with dominant $x \rightarrow e^{-} e^{+} \nu$ visible decays are constrained by Eqs.(6)-(13),(17); here the mixing parameters $\left|U_{\mu x}\right|$ and $\left|U_{e x}\right|$ are adjusted to give compatible values of branching fraction $B$ and visible-mode lifetime $\tau$ in Fig.1 (while $\left|U_{\tau x}\right|$ is negligible). Alternative solutions with dominant $x \rightarrow \nu \gamma$ visible decays are constrained by Eqs.([6),(16),([18)-([19); here $B$ and $\tau$ are determined by $\left|U_{\mu x}\right|$ and $\left|U_{\tau x}\right|$ instead (while $\left|U_{e x}\right|$ is negligible). Such interpretations imply that other sources of $x$-production should contribute appreciable additional $x$-decay events in the KARMEN detector, with different time-structures; in channels (a) and (b) the event rate depends on $\left|U_{e x}\right|$ and is appreciable in some Dirac $x \rightarrow e^{-} e^{+} \nu$ solutions and some $x \rightarrow \nu \gamma$ solutions, but in channel (c) the event rate is always $40 \%$ of the anomaly event rate. Similar $x$-decay signals should also appear in the LSND detector [2]. Since both detectors have approximately the same density, and since both $\nu$-interaction and $x$-decay events have the same inverse-square dependence on distance $L$ (so long as $L<<\beta_{x} \gamma_{x} \tau_{x} c$ ), the ratio of interactions to decays should be approximately the same in both experiments. Such $x$ decays could conceivably provide a background to the $\bar{\nu}_{\mu} \rightarrow \bar{\nu}_{e}$ oscillation search currently under way [3], although the $\bar{\nu}_{e} p \rightarrow e^{+} n$ signal is distinguishable in principle by detecting the delayed $2.2 \mathrm{MeV}$ gamma from the subsequent neutron capture $n(p, d) \gamma$. In high-energy neutrino beams, however, where the parent pions have been boosted to energies $E_{\pi}=\gamma m_{\pi}$ with $\gamma>>1$, the number of $x$-decays in a given detector volume will scale down as $p_{x}^{*} /\left[E_{x}^{*} \gamma\right]$ (where $p^{*}$ and $E^{*}$ are $\pi$-restframe momentum and energy) while the $\nu$ interactions will scale up as $\gamma$; thus the fraction that are decays decreases as $1 / \gamma^{2}$ and rapidly becomes negligible. Hence $x \rightarrow e^{+} e^{-} \nu$ decays cannot contribute significantly to the apparent $e / \mu$ excess in atmospheric neutrino events [35], where the parent pions and kaons have energies of 
$\mathcal{O}(\mathrm{GeV})$. Similarly, there should be a negligible $x \rightarrow e^{+} e^{-} \nu$ contribution in accelerator experiments such as BNL-E776 [36] which set an upper limit on $\nu_{\mu} \rightarrow \nu_{e}$ and $\bar{\nu}_{\mu} \rightarrow \bar{\nu}_{e}$ oscillations using $\mathrm{GeV}$ neutrinos.

Finally, we note that isosinglet (sterile) neutrinos occur naturally in $S O(10)$ and $E_{6}$ GUT models, as members of the basic fermion families [19]. A very light $\left(\approx 10^{-2} \mathrm{eV}\right)$ sterile neutrino has been suggested as a possible [37] or even necessary [38 participant in solar neutrino oscillations, while one with a mass of $\mathcal{O}(\mathrm{keV})$ is a good candidate for 'warm' dark matter [39]. Heavy singlet neutrinos could cause distinctive lepton-numbernonconserving, lepton-flavour-changing and lepton-universality-breaking effects in a wide range of laboratory processes 40].

\section{Acknowledgments}

RJNP thanks J. Kleinfeller, I. Blair and J. Guy for conversations; VB thanks R. Imlay for a discussion; SS thanks N. Booth, R. Mohapatra and G. Raffelt for helpful comments. This research was supported in part by the U.S. Department of Energy under Grant No. DE-FG02-95ER40896 and in part by the University of Wisconsin Research Committee with funds granted by the Wisconsin Alumni Research Foundation. SS is a PPARC Advanced Fellow and acknowledges support from the EC Theoretical Astroparticle Network.

\section{References}

[1] KARMEN collaboration: B. Armbruster et al, Phys. Lett. B348 (1995) 19.

[2] R.A. Reeder et al, Nucl. Instr. and Meth. A334 (1993) 353.

[3] D.O. Caldwell, in Trends in Astroparticle Physics, Stockholm 1994, eds. L. Bergström et al, Nucl. Phys. B, Proc. Suppl. (in press).

[4] A. De Rujula and S.L. Glashow, Phys. Rev. Lett. 45 (1980) 942;

P.B. Pal and L. Wolfenstein, Phys. Rev. D25 (1982) 766;

M. Gronau and R. Yahalom, Phys. Rev. D30 (1984) 2422.

[5] A. Hime, R.J.N. Phillips, G.G. Ross and S. Sarkar, Phys. Lett. B260 (1991) 381. 
[6] Particle Data Group, Phys. Rev. D50 (1994) 1173.

[7] R.E. Shrock, Phys. Rev. D24 (1981) 1232, 1275 .

[8] CHARM collaboration: F. Bergsma et al, Phys. Lett. 128B (1983) 361.

[9] BEBC-WA66 collaboration: A.M. Cooper-Sarkar et al, Phys. Lett. 160B (1985) 207.

[10] PS-191 collaboration: G. Bernardi et al, Phys. Lett. 166B (1986) 479.

[11] N. De Leener-Rosier et al, Phys. Rev. D43 (1991) 3611;

T. Numao et al, Proc. Beyond the Standard Model III, Ottawa, eds. S. Godfrey and P. Kalyniak (World Scientific, Singapore, 1993);

G. Czapek et al, Phys. Rev. Lett. 70 (1993) 17;

D.I. Britton et al, Phys. Rev. D49 (1994) 28.

[12] T. Bernatowicz et al, Phys. Rev. Lett. 69 (1992) 2341;

A. Balysh et al, Phys. Lett. B283 (1992) 32, preprint hep-ex/9502007;

J.-C. Vuilleumier et al, Phys. Rev. D48 (1993) 1009.

[13] P. Bamert, C.P. Burgess and R.N. Mohapatra, Nucl Phys. B438 (1995) 3.

[14] S. Petcov, Sov. J. Nucl. Phys. 25 (1977) 344, (Erratum) 25 (1978) 698;

B.W. Lee, S. Pakvasa, H. Sugawara and R.E. Shrock, Phys. Rev. Lett. 38 (1977) 937 ;

T.-P. Cheng and L.-F. Li, Phys. Rev. D16 (1977) 1425;

W.J. Marciano and A. Sanda, Phys. Lett. 67B (1977) 303.

[15] ARGUS collaboration: H. Albrecht et al, Phys. Lett. B292 (1992) 221.

[16] CLEO II collaboration: D. Cinabro et al, Phys. Rev. Lett. 70 (1993) 3700.

[17] ALEPH collaboration: D. Buskulic et al, preprint CERN PPE/95-003.

[18] The LEP Collaborations: preprint CERN PPE/94-187.

[19] G.B. Gelmini and E. Roulet, preprint UCLA/94/TEP/36 hep-ph/9412278];

R.N. Mohapatra and P.B. Pal, Massive Neutrinos in Physics and Astrophysics (World Scientific, 1991). 
[20] D.A. Dicus, E.W. Kolb and V.L. Teplitz, Astrophys. J. 221 (1978) 327;

E.W. Kolb and T. Goldman, Phys. Rev. Lett. 43 (1979) 897.

[21] S. Sarkar and A.M. Cooper, Phys. Lett. 148B (1984) 347.

[22] D. Lindley, Astrophys. J. 294 (1985) 1.

[23] S. Sarkar, Proc. XVII Int. Symp. on Multiparticle Dynamics, Seewinkel, eds. M. Markytan et al(World Scientific, 1986) p 863;

L.M. Krauss, Proc. Telemark Minconference on Neutrino Masses (1985).

[24] E.W. Kolb and R.J. Scherrer, Phys. Rev. D25 (1982) 1481;

N. Terasawa, M. Kawasaki and K. Sato, Nucl. Phys. 302 (1988) 697;

S. Dodelson, G. Guyk and M.S. Turner, Phys. Rev. D49 (1994) 5068.

[25] A. Dar and S. Dado, Phys. Rev. Lett. 59 (1987) 2368;

L. Oberauer, C. Hagner, G. Raffelt and E. Rieger, Astropart. Phys. 1 (1993) 377;

R.N. Mohapatra, S. Nussinov and X. Zhang, Phys. Rev. D49 (1994) 3434.

[26] S.W. Falk and D.N. Schramm, Phys. Lett. B79 (1978) 511;

R. Cowsik, D.N. Schramm and P. Höflich, Phys. Lett. B218 (1989) 91.

[27] G. Sigl and M.S. Turner, Phys. Rev. D51 (1995) 1499.

[28] G. Raffelt and G. Sigl, Astpart. Phys. 1 (1993) 165;

X. Shi and G. Sigl, Phys. Lett. B323 (1994) 360.

[29] K. Kainulainen, J. Maalampi and J.T. Peltoniemi, Nucl. Phys. B358 (1991) 435;

B. Mukhopadhyaya and R. Gandhi, Phys. Rev. D46 (1992) 3682.

[30] G. Raffelt, private communication.

[31] A. Manohar, Phys. Lett. B186 (1987) 370;

R. Barbieri and A. Dolgov, Nucl. Phys. B349 (1991) 743;

K. Enqvist, K. Kainulainen and M. Thompson, Nucl. Phys. B373 (1992) 498;

X. Shi, D.N. Schramm and B.D. Fields, Phys. Rev. D48 (1993) 2563.

[32] P. Langacker, B. Sathiapalan and G. Steigman, Nucl. Phys. B266 (1986) 669. 
[33] BEBC-WA66 collaboration: A.M. Cooper-Sarkar, private communication (quoted by K.S. Babu et al, Phys. Lett. B321 (1994) 140).

[34] V. Barger, J. Ohnemus and R.J.N. Phillips, Phys. Rev. D35 (1987) 166.

[35] K.S. Hirata et al, Phys. Lett. B280 (1992) 146;

Y. Fukuda et al, Phys. Lett. B335 (1994) 237.

[36] L. Borodovsky et al, Phys. Rev. Lett. 68 (1992) 274.

[37] V. Barger et al, Phys. Rev. D43 (1991) 1759;

C.-S. Lim and W.J. Marciano, Phys. Rev. D37 (1988) 1368.

[38] G.M. Fuller, J. Primack and Y.-Z. Qian, preprint DOE-ER-40561-185 astroph/9502081.

[39] S. Dodelson and L.M. Widrow, Phys. Rev. Lett. 72 (1994) 17.

[40] see P. Langacker, L. Luo and A.K. Mann, Rev. Mod. Phys. 64 (1992) 87;

J. Bernabeu et al, Phys. Rev. Lett. 71 (1993) 2695;

A. Ilakovac and A. Pilaftsis, Nucl. Phys. B437 (1995) 491;

P. Roy, preprint TIFR-TH-94-35 hep-ph/9501209; and references therein.

\section{Figures}

1. Correlation between the $x$ mean lifetime and production branching ratio $B=$ $\Gamma(\pi \rightarrow \mu x) / \Gamma(\pi \rightarrow \mu \nu)$, needed to explain the KARMEN anomaly; the solid curve is taken from Ref. [1] and the dashed curve is its extrapolation. The $\left|U_{\mu x}\right|^{2}$ scale is derived from Eq.(4) for $B$. The $\left|U_{e x}\right|^{2}$ scale is derived from Eq.(5) for the case of dominant $x \rightarrow e^{-} e^{+} \nu$ visible decays with Dirac $x$. The most stringent laboratory constraints on the $\left|U_{\alpha x}\right|$ values are also shown [6, [, 10,11]. For Majorana $x$ there is an additional constraint $\left|U_{e x}\right|^{2} \lesssim 6 \times 10^{-8}$ and the $\left|U_{e x}\right|^{2}$ scale moves left by a factor of 2 .

2. Visible energy spectra for $x \rightarrow$ eev decays from various sources, weighted by the inverse of the $x$ momentum for the relative decay probability in a given detector. 
The solid curve denotes the stopped $\pi^{+} \rightarrow \mu^{+} x$ source; dotted, dashed, and dotdashed curves denote the $\pi^{+} \rightarrow e^{+} x, \pi^{+} \rightarrow \mu^{+} \rightarrow \bar{\nu}_{\mu} x e^{+}$and $\pi^{+} \rightarrow \mu^{+} \rightarrow \bar{x} \nu_{e} e^{+}$ sources, respectively. 


\section{Erratum}

In the case that neutrino $x$ is mainly isosinglet (sterile), we overlooked neutral-current contributions to the decay $x \rightarrow \nu e^{+} e^{-}$, as pointed out by J. Peltoniemi (hep-ph/9606228). The corrected width for small mixing is

$\Gamma\left(x \rightarrow \nu e^{+} e^{-}\right)=390 K\left[\left(1+4 x_{W}+8 x_{W}^{2}\right)\left|U_{e x}\right|^{2}+\left(1-4 x_{W}+8 x_{W}^{2}\right)\left(\left|U_{\mu x}\right|^{2}+\left|U_{\tau x}\right|^{2}\right)\right] \mathrm{s}^{-1}$, where $x_{W} \equiv \sin ^{2} \theta_{W}=0.23, K=1(2)$ for Dirac (Majorana) $x$ and the $\left|U_{e x}\right|^{2}$ term includes charged-current contributions. The visible decay width (including $x \rightarrow \nu \gamma$ ) and total width then become

$$
\begin{aligned}
& \Gamma_{\text {vis }}=K\left[920\left|U_{e x}\right|^{2}+210\left|U_{\mu x}\right|^{2}+210\left|U_{\tau x}\right|^{2}\right] \mathrm{s}^{-1}, \\
& \Gamma_{\text {tot }}=K\left[2470\left|U_{e x}\right|^{2}+1760\left|U_{\mu x}\right|^{2}+1760\left|U_{\tau x}\right|^{2}\right] \mathrm{s}^{-1} .
\end{aligned}
$$

The KARMEN event rate determines the product $B\left(\pi^{+} \rightarrow \mu^{+} x\right) \Gamma_{\text {vis }} \simeq 3 \times 10^{-11} \mathrm{~s}^{-1}$ with $B=0.0285\left|U_{\mu x}\right|^{2}$ as before, which allows different types of solution:

(A) If $\left|U_{e x}\right|^{2}$ dominates $\Gamma_{\text {vis }}$, we obtain $\left|U_{e x} U_{\mu x}\right|^{2} \simeq 1.1 K^{-1} \times 10^{-12}$. For Majorana $x$, no such solutions are compatible both with the constraint Eq.(9) and with $\left|U_{e x}\right|^{2}$

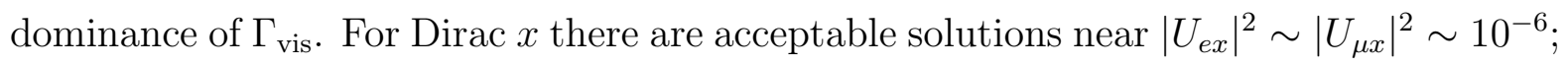
but as $\left|U_{e x}\right|$ decreases, $\left|U_{\mu x}\right|$ increases and they merge into category (B).

(B) If $\left|U_{\mu x}\right|^{2}$ dominates $\Gamma_{v i s}$, we obtain a fixed value $\left|U_{\mu x}\right|^{2} \simeq 2.3 K^{-1 / 2} \times 10^{-6}$. These solutions allow ranges of $\left|U_{e x}\right|,\left|U_{\tau x}\right|<\left|U_{\mu x}\right|$, but when $\left|U_{\tau x}\right|$ increases further they merge into category $(\mathrm{C})$.

(C) If $\left|U_{\tau x}\right|^{2}$ dominates $\Gamma_{\text {vis }}$, we obtain $\left|U_{\mu x} U_{\tau x}\right|^{2} \simeq 5 K^{-1} \times 10^{-12}$, with $1.5 K^{-1} \times$ $10^{-6}<\left|U_{\tau x}\right|^{2}<1$, where the upper (unitarity) limit cannot be approached closely because $x$ cannot be the main component of $\nu_{\tau}$. Solutions (A) and (B) give mean lifetimes $\tau_{x} \sim(150-300) \mathrm{s}$, but $(\mathrm{C})$ covers the range $\tau_{x} \sim(0.001-150) \mathrm{s}$.

The neutral-current contributions now guarantee that $x \rightarrow \nu e^{+} e^{-}$is always the dominant visible mode. Eqs.(16)-(17) are invalidated and some parameters change, but our qualitative conclusions about the existence of solutions (especially short-lived type-(C) cases) remain broadly unchanged.

Concerning additional sources of $x$ at KARMEN, the $\pi^{+} \rightarrow e x$ and $\mu^{+} \rightarrow \bar{\nu}_{\mu} x e^{+}$ channels depend on the ratio $\left|U_{e x} / U_{\mu x}\right|^{2}$ as before; this ratio is $\lesssim 1$ for (A) and (B) but can be large for type-(C) solutions. The $\mu^{+} \rightarrow \bar{x} \nu_{e} e^{+}$rate is fixed and unchanged. 
This figure "fig1-1.png" is available in "png" format from: http://arxiv.org/ps/hep-ph/9503295v4 


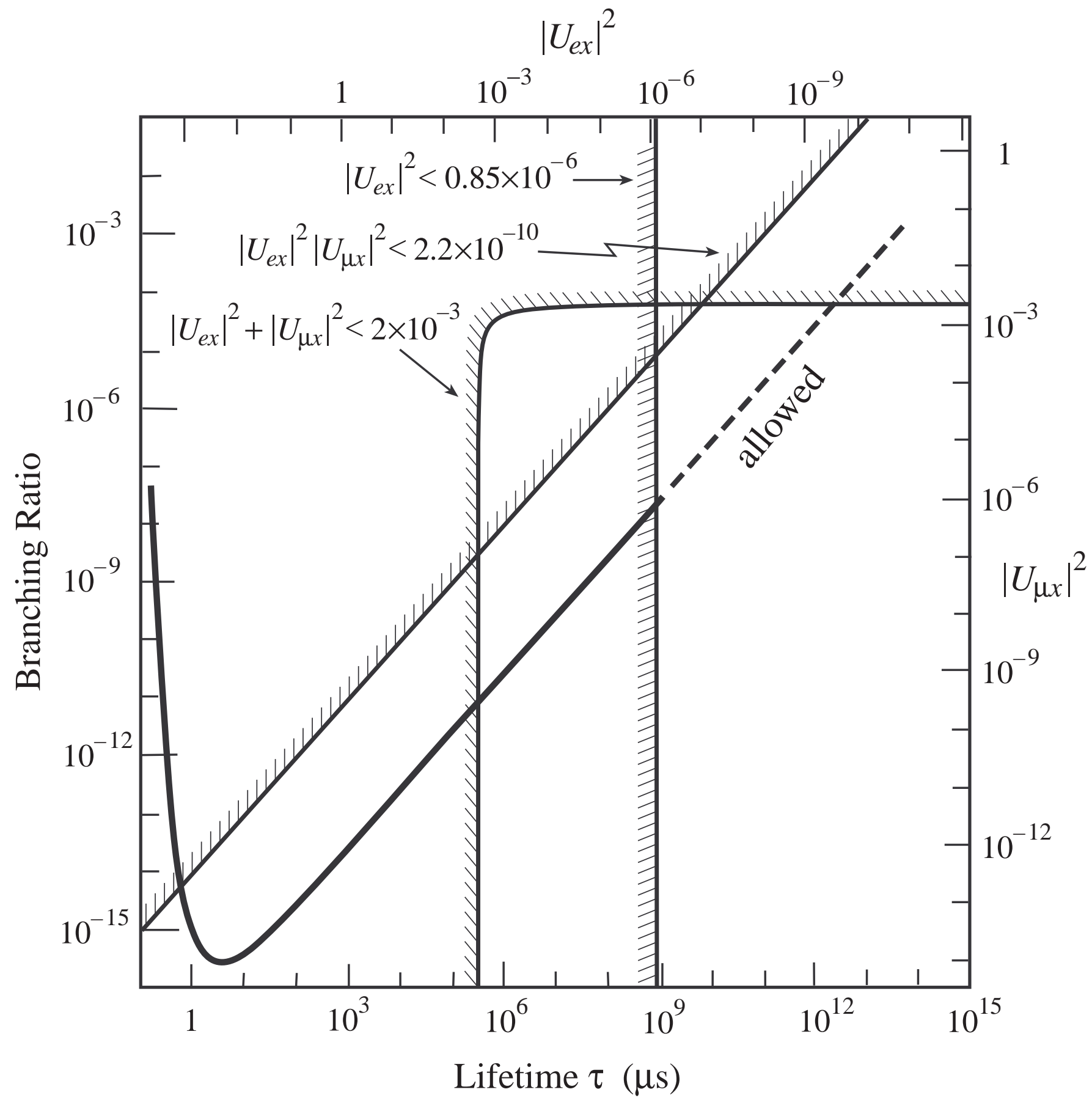

Figure 1 
This figure "fig1-2.png" is available in "png" format from: http://arxiv.org/ps/hep-ph/9503295v4 


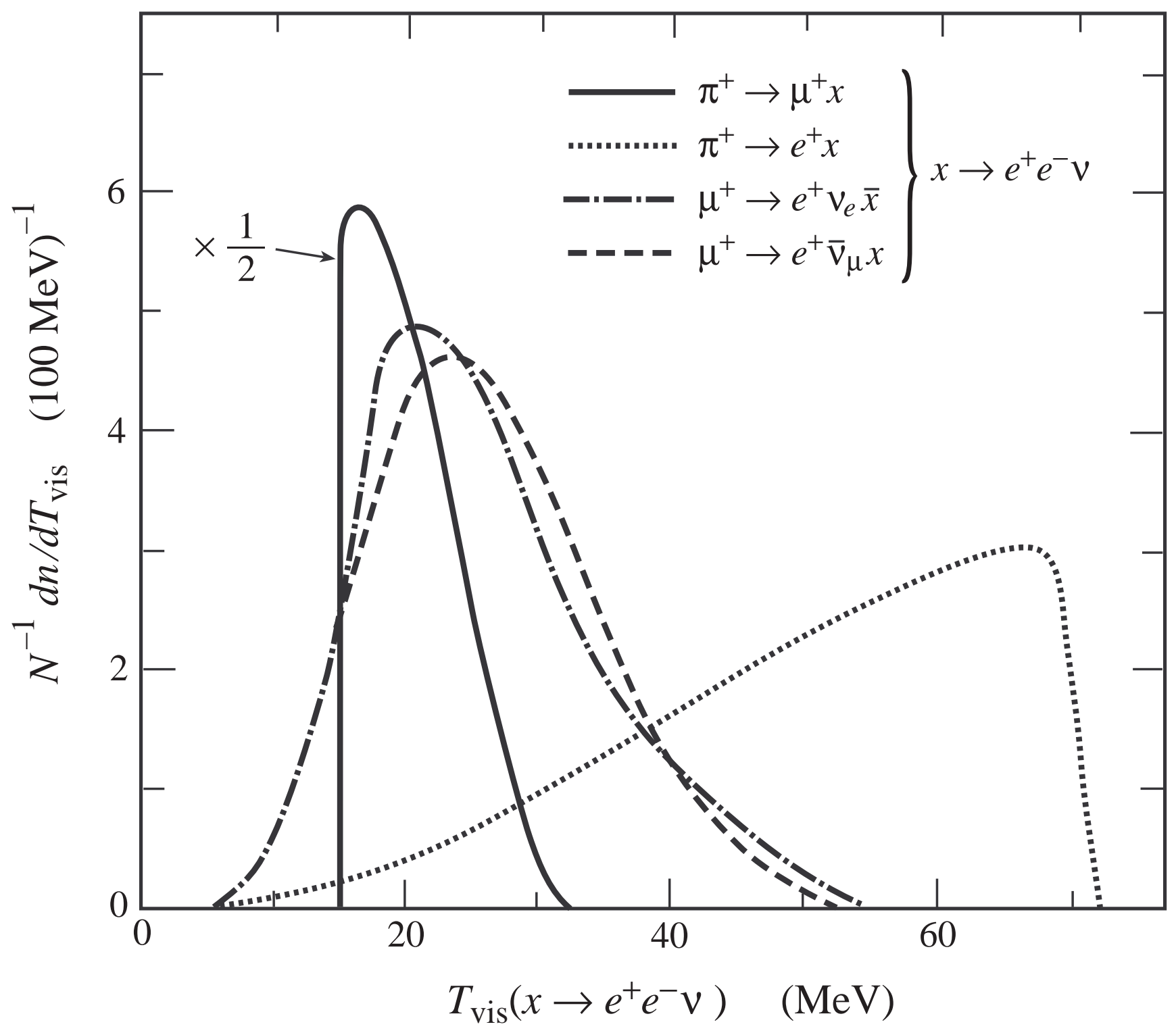

Figure 2 